\title{
Improving the Sharpness of Digital Images Using a Modified Laplacian Sharpening Technique
}

\author{
Zohair Al-Ameen ${ }^{1}$, Shamil Al-Ameen ${ }^{2}$, Ahmed Al-Othman ${ }^{3}$
}

\begin{abstract}
Many imaging systems produce images with deficient sharpness due to different real limitations. Hence, various image sharpening techniques have been used to improve the acutance of digital images. One of such is the well-known Laplacian sharpening technique. When implementing the basic Laplacian technique for image sharpening, two main drawbacks were detected. First, the amount of introduced sharpness cannot be increased or decreased. Second, in many situations, the resulted image suffers from a noticeable increase in brightness around the sharpened edges. In this article, an improved version of the basic Laplacian technique is proposed, wherein it contains two key modifications of weighting the Laplace operator to control the introduced sharpness and tweaking the second order derivatives to provide adequate brightness for recovered edges. To perform reliable experiments, only real-degraded images were used, and their accuracies were measured using a specialized no-reference image quality assessment metric. From the obtained experimental results, it is evident that the proposed technique outperformed the comparable techniques in terms of recorded accuracy and visual appearance.
\end{abstract}

Keywords-Image sharpening; Laplace operator; Modified Laplacian; Second-order derivatives;

\section{INTRODUCTION}

$\mathrm{D}$ igital images have dissimilar edge profiles due to the nature of objects placed into the captured scene. Specifically, contours, edges and local details are specific image components that hold important information which is significant to the observer and the apparent quality of the image. However, such information may not appear sharp due to certain inevitable limitations of the imaging equipment. Thus, the digital image degrades in quality and appears blurry (unsharp) [5]. Image sharpening techniques are usually used in digital image processing to increase the visual appearance of weak contours, edges and local details. The foremost aim of image sharpening is to enhance the quality of a given image in a way that it appears clearer and sharper in terms of visible contours and edges [4].

For this purpose, a great variety of image sharpening techniques have been introduced, including Laplacian [7], unsharp mask, [8], nonlinear diffusion equation [9], contourlet transform [10], hyperbolic secant filtering [11], fuzzy logic [12], shock filtering [13], morphological filtering [14], and many more. Each of the aforementioned techniques has performance limitations in addition to specific hardware and software requirements. Moreover, their complexity varies from low to high due the use of various processing concepts. One noncomplex concept for image sharpening is the use of first and second-order derivatives [4]. The Laplacian, which is a second-order derivative, has been widely used in many sharpening related applications [6].

The Laplacian technique simply sharpens an image by

Zohair Al-Ameen is with the Department of Computer Science, College of Computer Science and Mathematics, University of Mosul, Nineveh, Iraq. E-mail: qizohair@gmail.com

Shamil Al-Ameen is with the Department of Software Engineering, College of Computer Science and Mathematics, University of Mosul, Nineveh, Iraq. E-mail: shamilqays@gmail.com

Ahmed Al-Othman is with the Department of Computer Science, College of Education for Pure Sciences, University of Mosul, Nineveh, Iraq. E-mail: ahmedbasi10081@gmail.com emphasizing the high gradient information in an image. In this study, and due to its low-cost nature, the focus was mainly on the development of uncomplicated postprocessing technique to mitigate the blur effect. That's why the basic Laplacian technique was selected to be improved. When implementing this technique for image sharpening, two main drawbacks were detected. First, the amount of introduced sharpness cannot be increased or decreased. Second, in many situations, the resulted image suffers from a noticeable increase in brightness around the sharpened edges. These undesirable drawbacks must be resolved to ameliorate the performance of image sharpening using a noncomplex technique.

Thus, an improved version of the basic Laplacian technique is proposed in this study, in which it contains two key modifications of weighting the Laplace operator to control the introduced sharpness and tweaking the second order derivatives to provide satisfactory brightness for recovered edges, as well as allow a better control of the sharpening process. To perform reliable experiments, real-degraded images were used only for experimental and comparison purposes. Moreover, the accuracies of the attained results were measured using specialized no-reference image quality assessment metric. The rest of this article is organized as follows: In Section II, an adequate explanation regarding the proposed technique is provided. In Section III, the results and the required discussions are delivered. Finally, a concise conclusion is given in Section IV.

\section{PRoposed TECHNIQUE}

To improve the acutance of a given image, second order derivatives are usually used over first order derivatives, because they are more sensitive to intensity variations. In addition, the Laplace operator which is a second order derivative is well-known for being used in many image sharpening applications [6]. In order to implement a basic Laplacian sharpening technique, three essential phases are required. The first phase involves the calculation of the second order derivatives for a given 
image using Eq. $(1,2)[1]$.

$$
\begin{aligned}
& \frac{\partial^{2} f(x, y)}{\partial x^{2}}=f(x+1, y)-2 f(x, y)+f(x-1, y) \\
& \frac{\partial^{2} f(x, y)}{\partial y^{2}}=f(x, y+1)-2 f(x, y)+f(x, y-1)
\end{aligned}
$$

where, $f(x, y)$ represents the inputted image at the real coordinate position $(x, y)$. The second phase involves the calculation of the Laplace operator $\nabla^{2} f(x, y)$ using Eq. (3) $[2]$.

$$
\nabla^{2} f(x, y)=\frac{\partial^{2} f(x, y)}{\partial x^{2}}+\frac{\partial^{2} f(x, y)}{\partial y^{2}}
$$

The third phase involves the determination of the sharp image $k(x, y)$ using Eq. (4) [3].

$$
k(x, y)=f(x, y)-\nabla^{2} f(x, y)
$$

However, this technique suffers from two main drawbacks. Firstly, the amount of introduced sharpness cannot be controlled (i.e. increased or decreased). Secondly, in many situations, the resulted image suffers from a noticeable increase in brightness around its sharpened edges. These undesirable drawbacks must be resolved to ameliorate the performance of image sharpening. Hence, two modifications are proposed in this study to overcome aforementioned drawbacks. The first modification includes tweaking the second order derivatives in Eq. (1, 2), which helps in providing acceptable brightness for recovered edges and allowing a better control of the overall sharpening process. The tweaked derivatives are computed using Eq. $(5,6)$.

$$
\begin{aligned}
& \frac{\partial^{2} f(x, y)}{\partial x^{2}}=[\Upsilon(f(x+1, y)-2 f(x, y)+f(x-1, y))]^{\Gamma} \\
& \frac{\partial^{2} f(x, y)}{\partial y^{2}}=[\Upsilon(f(x, y+1)-2 f(x, y)+f(x, y-1))]^{\Gamma}
\end{aligned}
$$

where, $\Upsilon$ and $\Gamma$ are two tweaking parameters that are set by default to 0.1 and 0.75 , respectively. The parameter $\Upsilon$ allows a better sharpness control when using the weighted Laplace operator, while parameter $\Gamma$ allows the production of acceptable brightness for recovered edges. The second modification includes the inclusion of a distinct weighting parameter to the Laplace operator in Eq. (4) to improve its processing performance. The weighted Laplace operator, which is developed in this study, is computed using Eq. (7).

$$
\nabla^{2} f(x, y)=\Lambda\left[\frac{\partial^{2} f(x, y)}{\partial x^{2}}+\frac{\partial^{2} f(x, y)}{\partial y^{2}}\right]
$$

where, $\Lambda$ is a weighting parameter that is used to control the apparent sharpness. This parameter should fulfill $(\Lambda>1)$, in which a higher $\Lambda$ value leads to sharper result and vice versa. In order to obtain the proposed sharpening technique, Eq. (1) to Eq. (3) are replaced by their modified counterparts of Eq. (5) to Eq. (7). It is important to mention that the achieved modifications are determined based on intensive experiments on various degraded images. To end with, the following pseudocode in Technique 1 is provided to support an accurate description regarding the execution specifics of the proposed technique.

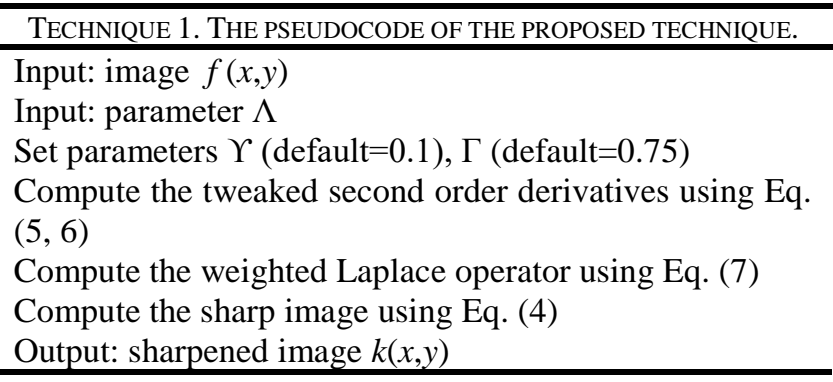

\section{RESULTS AND DISCUSSION}

In this section, the necessary computer experiments and evaluations are provided to exhibit and appraise the performance of the proposed technique with several comparable techniques, as well as various real-degraded images. Accordingly, different techniques for comparison were selected including, the classical shock filter [16], classical unsharp mask filter [8], 8 neighborhood operator [12], in addition to the basic Laplacian technique. Moreover, the dataset of images used in this study were collected for the overtly available digital image databases. It is important to measure the quality of the obtain results using an impartial metric. Thus, may specialized no-reference image quality metrics were studied, for such the cumulative probability of blur detection (CPBD) [15] was selected due to its suitability with the nature of the studied problem.

Accordingly, the CPBD metric was developed based on the human blur perception by estimating the probability of the identified blur at every edge in the image. Afterwards, the previously collected information is combined by calculating the CPBD. The input to the CPBD metric is a single image, and the output is a numerical value which is higher than 0 , in which a higher value means that the inputted image is sharper in attributes. Figure 1 - Figure 3 exhibit the results of processing real-degraded images by the proposed technique. Figure 4 and Figure 5 demonstrate the comparison results. Table 1 displays the recorded accuracy by the CPBD metric for the comparative techniques. Figure 6 shows an analytical chart of the recorded accuracy by the CPBD metric.

From the obtained results displayed in Figure 1 Figure 3, it can be seen that the visual qualities of the results obtained by the proposed technique are better than those obtained by the basic technique, and way better than the quality of the original images. Accordingly, it provided visually pleasing results that have a natural appearance, acceptable brightness for recovered edges, and no visible processing flaws. Regarding the comparison results which are exhibited in Figure 4 - Figure 6 and Table 1, it can be seen that the proposed technique outperformed the comparable technique in terms of visual quality and recorded accuracy as it scored the highest according to the CPBD metric and it provided the best perceived quality with no apparent flaws.

However, although the shock filter provided sharp edges, it provided low CPBD measures and produced the unwanted staircase effect. This effect made the output of this method unacceptable in many real applications. Moreover, the unsharp mask filter it delivered reasonable improvement for fine image details as identified by the CPBD metric. However, it presented the overshoot 
effect, which is deemed undesirable for many image processing applications. In addition, processing the images by the 8 neighborhood operator led to deficient quality results that are over-sharpened and has a noticeable increase in noise. However, it scored relatively high according to the CPBD metric because it provided high sharpening amount to the processed images. The introduced effects by this technique are unwanted for digital images. In the end, using low complexity techniques to improve the sharpness of digital images is desirable in many areas of digital image processing. Therefore, this study was conducted to provide a good technique which can be used in many real image processing applications.

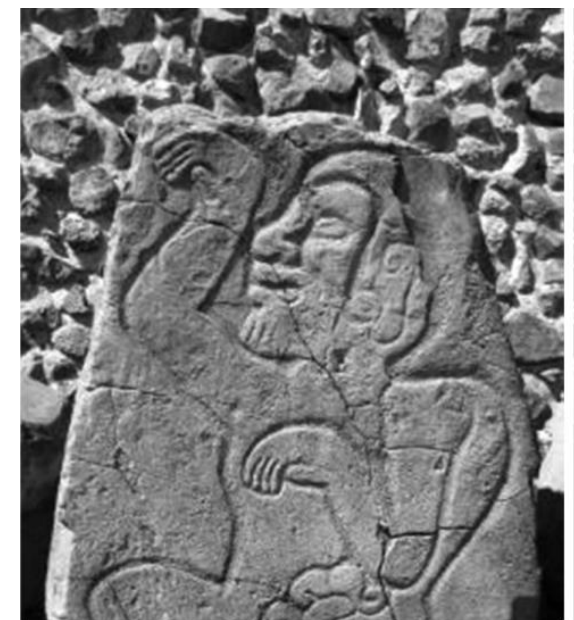

(a)

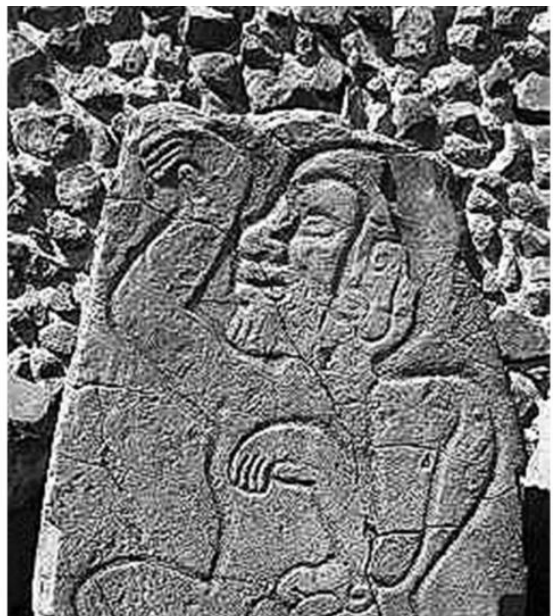

(b)

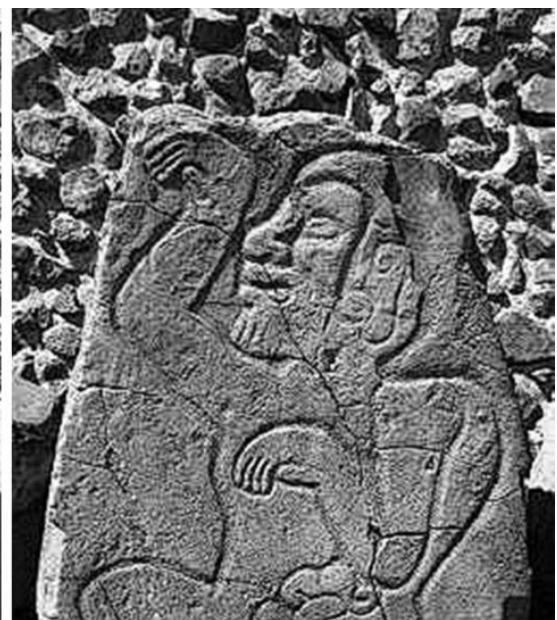

(c)

Figure. 1. Processing a real-degraded image: (a) unsharp image; (b) processed by the basic Laplacian technique; (c) processed by the proposed technique $(\Lambda=11)$.

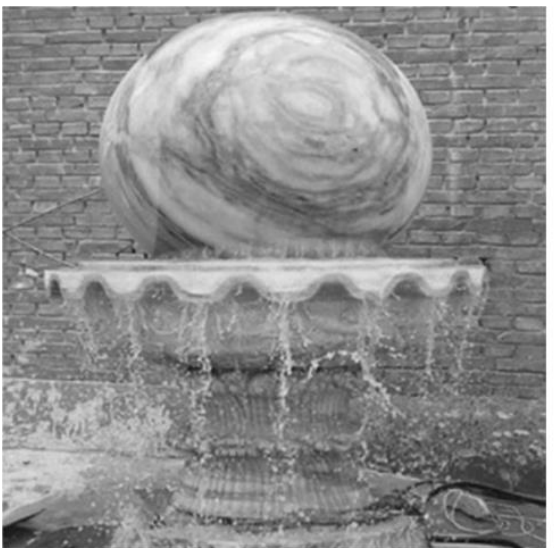

(a)

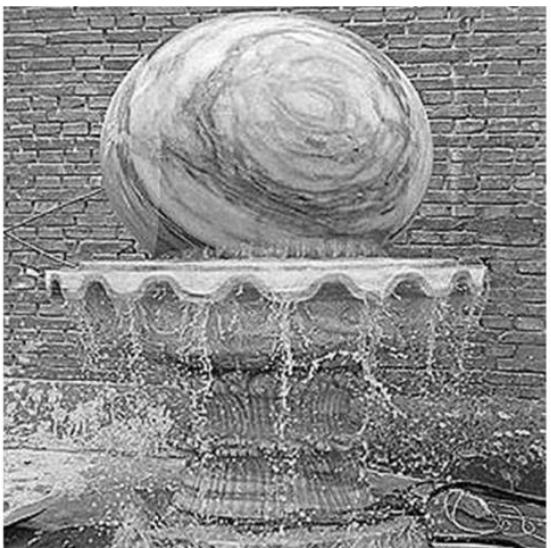

(b)

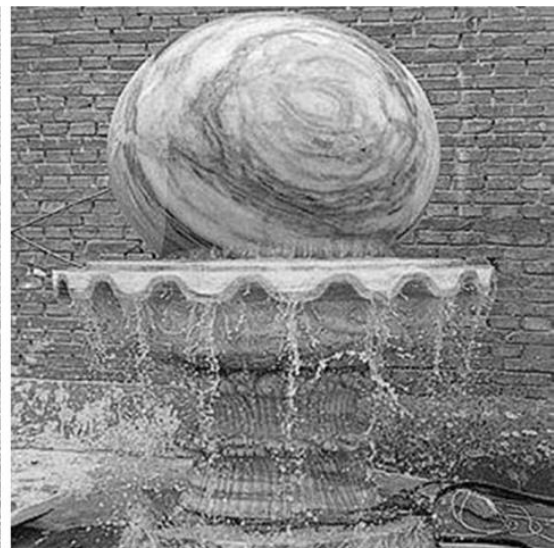

(c)

Figure. 2. Processing a real-degraded image: (a) unsharp image; (b) processed by the basic Laplacian technique; (c) processed by the proposed technique $(\Lambda=10)$.

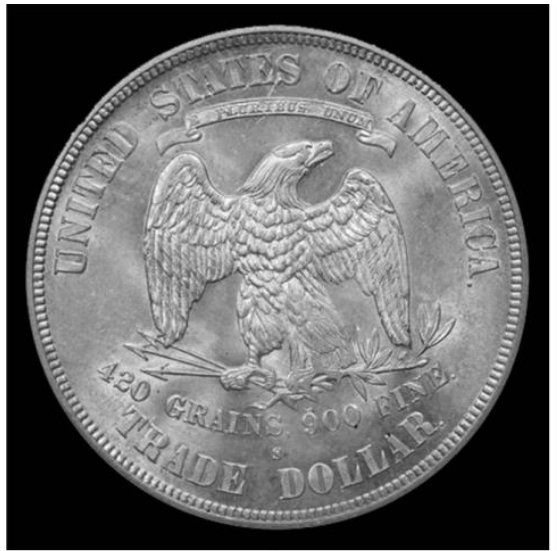

(a)

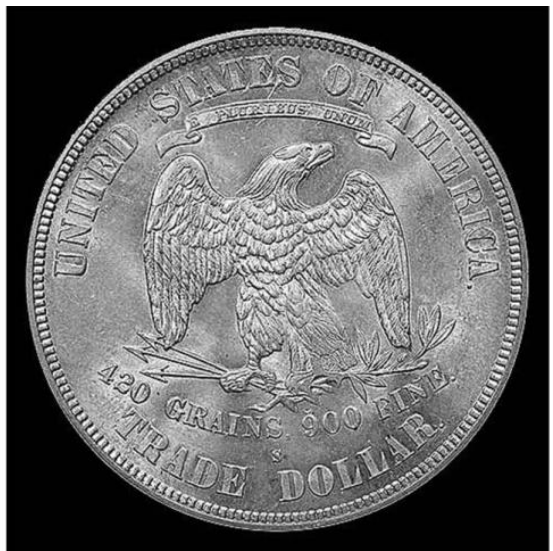

(b)

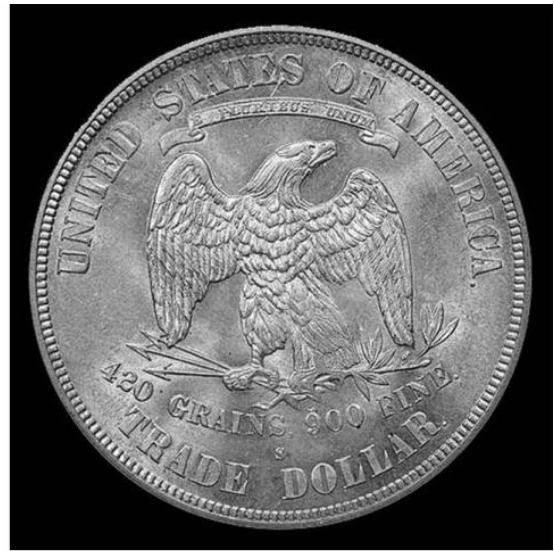

(c)

Figure. 3. Processing a real-degraded image: (a) unsharp image; (b) processed by the basic Laplacian technique; (c) processed by the proposed technique $(\Lambda=9)$. 


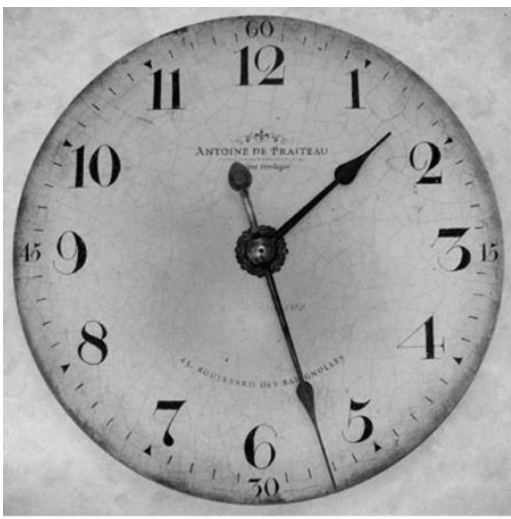

(a)

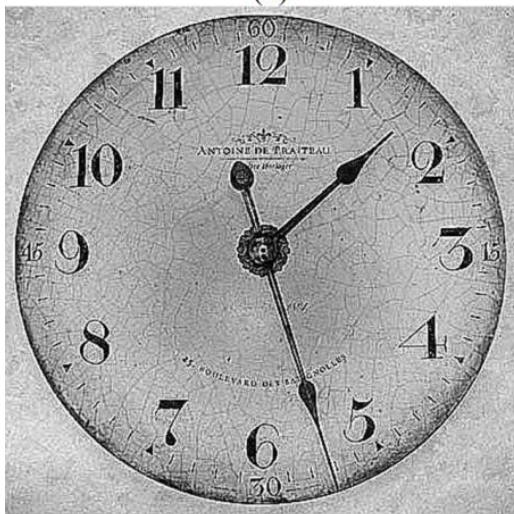

(d)

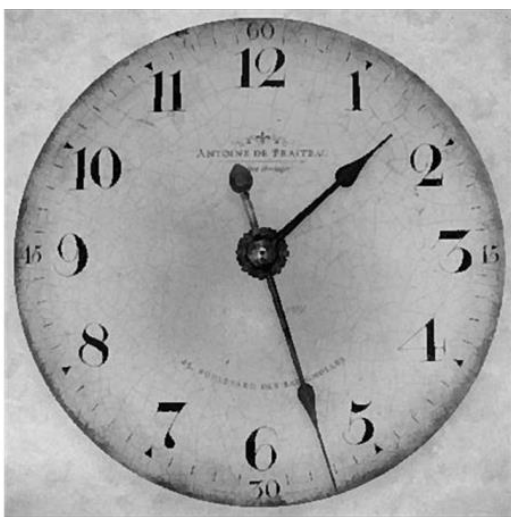

(b)

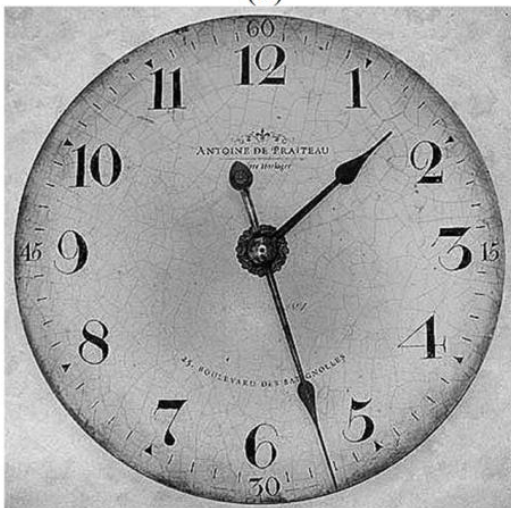

(e)

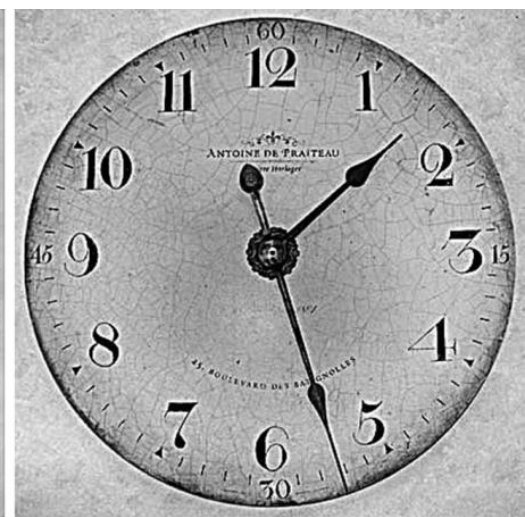

(c)

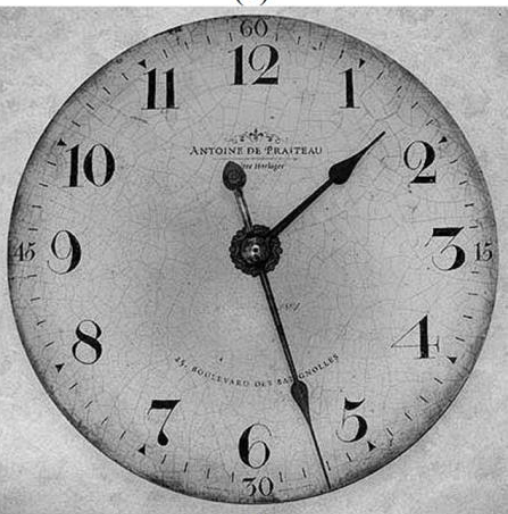

(f)

Figure. 4. Processing a real-degraded image by the proposed and the comparable techniques. (a) real-degraded image; the rest of the images are processed by: (b) classical shock filter; (c) classical unsharp mask filter; (d) 8 neighborhood operator; (e) basic Laplacian technique; (f) proposed technique $(\Lambda=10)$.

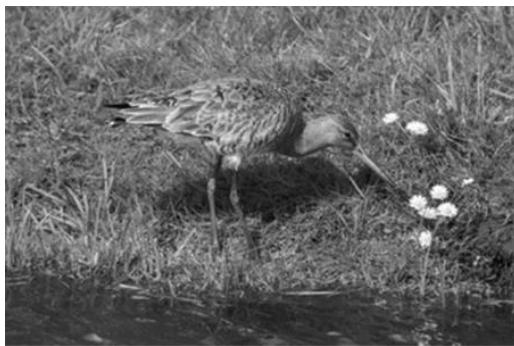

(a)

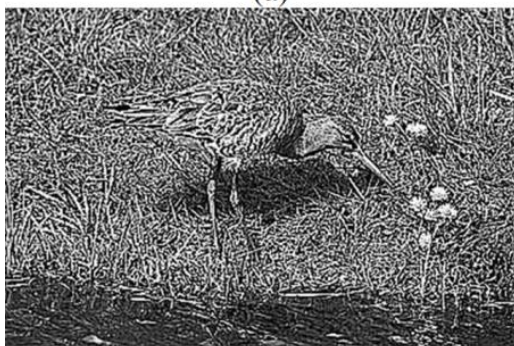

(d)

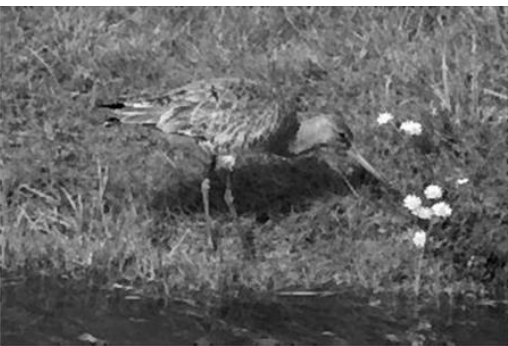

(b)

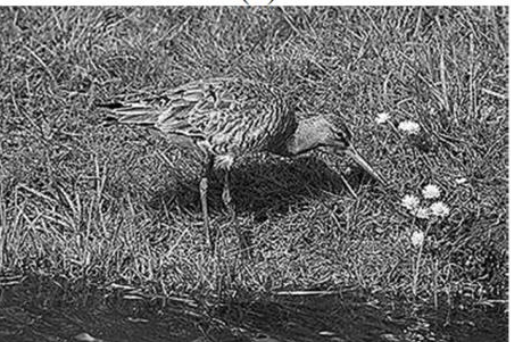

(e)

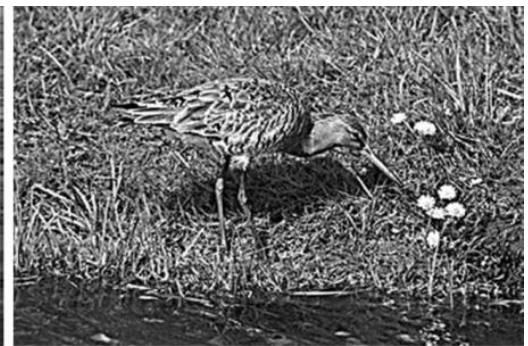

(c)

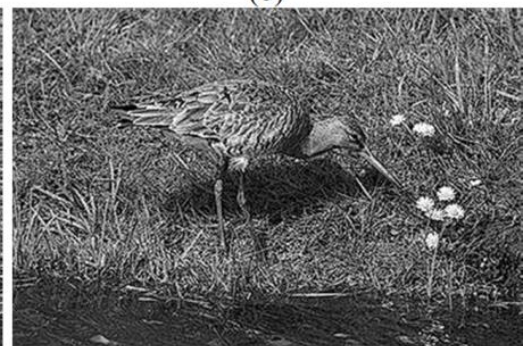

(f)

Figure. 5. Processing a real-degraded image by the proposed and the comparable techniques. (a) real-degraded image; the rest of the images are processed by: (b) classical shock filter; (c) classical unsharp mask filter; (d) 8 neighborhood operator; (e) basic Laplacian technique; (f) proposed technique $(\Lambda=13)$.

\begin{tabular}{llcc}
\multicolumn{4}{l}{ TABLE 1. THE RECORDED ACCURACY BY THE CPBD METRIC FOR THE COMPARATIVE TECHNIQUES } \\
\hline$\#$ & Comparatives & Figure 4 & Figure 5 \\
\hline 1 & Original Image & 0.7927 & 0.8095 \\
2 & Classical Shock Filter & 0.8242 & 0.7703 \\
3 & Classical Unsharp Mask Filter & 0.8011 & 0.8480 \\
4 & 8 Neighborhood Operator & 0.8657 & 0.9064 \\
5 & Basic Laplacian Technique & 0.8685 & 0.9082 \\
6 & Proposed Technique & $\mathbf{0 . 8 6 9 2}$ & $\mathbf{0 . 9 0 9 1}$ \\
\hline
\end{tabular}

Note: the bold values indicate the best achieved results. 


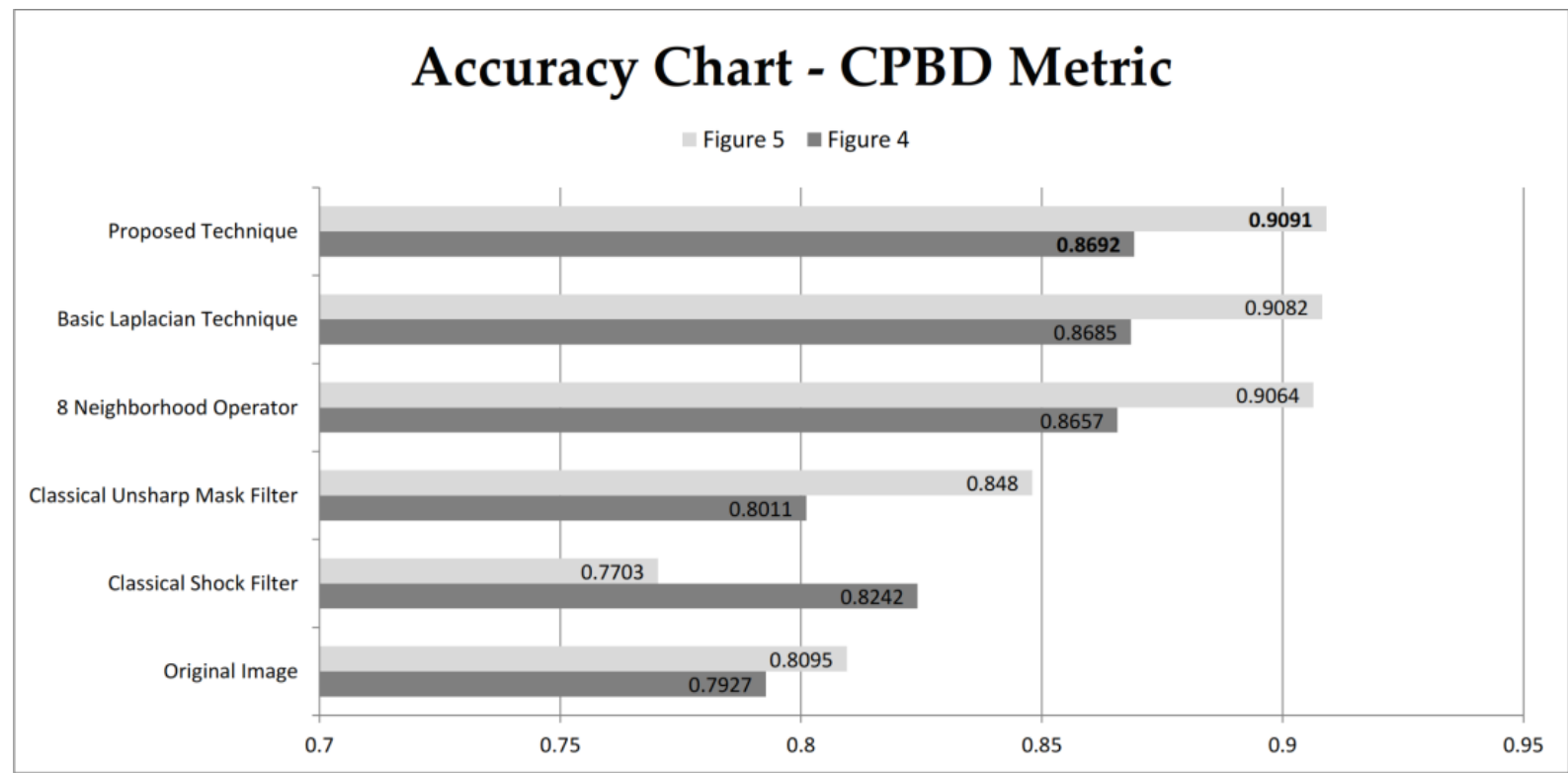

Figure. 6. The analytical chart of the recorded accuracy values using a CPBD metric.

\section{CONCLUSION}

The use of the Laplacian technique to improve the acutance of digital images is widely known. Thus, a modified version of the aforesaid technique is proposed in this study, in which it contains two key modifications of weighting the Laplace operator to control the introduced sharpness and tweaking the second order derivatives to provide satisfactory brightness for recovered edges, as well as allow a better control of the sharpening process. Regarding the conducted experiments, only real-degraded images were used, and the accuracy were measured using an advanced noreference metric. From the obtained results, it can be seen the proposed technique produced satisfactory results in terms of recorded accuracy and perceived quality, as it outperformed the comparable techniques and scored the highest scores using a CPBD metric. In addition, the proposed technique provided natural looking images with no visible errors. Finally, the proposed technique is expected to be used in various image processing applications, especially those that require low calculations.

\section{ACKNOWLEDGEMENT}

The authors would like to thank the respectable editorial members and reviewers for their helpful remarks.

\section{REFERENCES}

[1] L. Juncheng, "Edge detection using 1 2-order fractional differential mask", Computer Engineering and Applications, vol. 50, no. 21, pp. 14-18, 2014.

[2] C. Dharmaraj, M. Krishna and R. Murugesan, "A Feature Identification System for Electron Magnetic Resonance Tomography: Fusion of Principal Components Transform, Color Quantization and Boundary Information", Journal of Mathematical Imaging and Vision, vol. 30, no. 3, pp. 284-297, 2008.
[3] H. Kotera and H. Wang, "Multiscale image sharpening adaptive to edge profile", Journal of Electronic Imaging, vol. 14, no. 1, pp. 013002-1-013002-17, 2005.

[4] Z. Afrose and Y. Shen, "Mesh color sharpening", Advances in Engineering Software, vol. 91, pp. 36-43, 2016.

[5] S. Fu, Q. Ruan, W. Wang, F. Gao and H. Cheng, "A featuredependent fuzzy bidirectional flow for adaptive image sharpening", Neurocomputing, vol. 70, no. 4-6, pp. 883-895, 2007.

[6] T. Ma, L. Li, S. Ji, X. Wang, Y. Tian, A. Al-Dhelaan and M. AlRodhaan, "Optimized Laplacian image sharpening algorithm based on graphic processing unit", Physica A: Statistical Mechanics and its Applications, vol. 416, pp. 400-410, 2014.

[7] M. Millán and E. Valencia, "Color image sharpening inspired by human vision models", Applied Optics, vol. 45, no. 29, pp. 7684 7697, 2006.

[8] K. Panetta, Y. Zhou, S. Agaian and H. Jia, "Nonlinear Unsharp Masking for Mammogram Enhancement", IEEE Transactions on Information Technology in Biomedicine, vol. 15, no. 6, pp. 918 928, 2011.

[9] D. Fang, Z. Nanning and X. Jianru, "Image smoothing and sharpening based on nonlinear diffusion equation", Signal Processing, vol. 88, no. 11, pp. 2850-2855, 2008.

[10]S. Anand, R. Shantha Selva Kumari, T. Thivya and S. Jeeva, "Sharpening enhancement of ultrasound images using contourlet transform", Optik - International Journal for Light and Electron Optics, vol. 124, no. 21, pp. 4789-4792, 2013.

[11]S. Anand and R. Shantha Selva Kumari, "Sharpening enhancement of Computed Tomography (CT) images using Hyperbolic Secant Square filter", Optik - International Journal for Light and Electron Optics, vol. 124, no. 15, pp. 2121-2124, 2013.

[12]Z. Gui and Y. Liu, "An image sharpening algorithm based on fuzzy logic", Optik - International Journal for Light and Electron Optics, vol. 122, no. 8, pp. 697-702, 2011.

[13]S. Bettahar and A. Stambouli, "Shock filter coupled to curvature diffusion for image denoising and sharpening", Image and Vision Computing, vol. 26, no. 11, pp. 1481-1489, 2008

[14]J. Schavemaker, M. Reinders, J. Gerbrands and E. Backer, "Image sharpening by morphological filtering", Pattern Recognition, vol. 33 , no. 6, pp. 997-1012, 2000.

[15]N. Narvekar and L. Karam, "A No-Reference Image Blur Metric Based on the Cumulative Probability of Blur Detection (CPBD)", IEEE Transactions on Image Processing, vol. 20, no. 9, pp. 2678-2683, 2011.

[16]S. Osher and L. Rudin, "Feature-oriented image enhancemen using shock filters", SIAM Journal on Numerical Analysis, vol. 27, no. 4, pp. 919-940, 1990. 